\title{
Wuhan's Experience in Fighting COVID-19: Achieving Zero Case of COVID-19 Infection among Healthcare Workers
}

\author{
Anhua $\mathrm{Wu}^{1,3}$, Liuyi Li ${ }^{2}$, Caixia Tan ${ }^{1}$, Xiujuan Meng ${ }^{1}$, Xudong $\mathrm{Ma}^{4}$, Chunhui Li ${ }^{1,3 *}$
}

1 Infection Center of Xiangya Hospital, Central South University, Changsha, Hunan Province, China 410008

2 Department of Hospital Infection Control, Peking University First Hospital, Beijing, China 100034

3 National Clinical Research Center for Geriatric Disorders (Xiangya Hospital), Changsha, Hunan Province, China 410008

4 Bureau of Medical Administration, National Health Commission of the People's Republic of China, Beijing, China 100044

* Correspondence: Chunhui Li, M.D, Infection Control Center, Xiangya Hospital, Central South University, Changsha, Hunan Province, China 410008. Tel: +86 731 89753953; Fax: +86 73184327237 E-mail: lichunhui@csu.edu.cn

\begin{abstract}
In the context of the COVID-19 pandemic, thousands of healthcare workers (HCWs) infected with COVID-19 have lost their lives worldwide. At the early stage of the epidemic, when COVID-19 was still not considered as a pandemic, a large number of Chinese HCWs were infected. Officials reported that more than 3,000 HCWs in Hubei contracted the virus at the early stage of the outbreak due to limited knowledge of the virus. Following reports of overloaded local hospitals, more than 42,000 medical staff, including those from the military, were dispatched to Hubei from across the country. At the peak of the fight, one in 10 intensive care medics in China were working in Wuhan. During fighting against COVID-19 in China, although a large number of HCWs were infected by SARS-CoV-2 in the early stages of the epidemic, the timely adoption of measures indicated that, a faster rate of diagnosis could be achieved, patients were isolated in-time, HCWs' safety was prioritized, training on basic protective knowledge and unified management of HCWs was strengthened, and effective protective measures were implemented. This resulted in the accomplishment of zero SARS-CoV-2 infection among the 42,632 members of the national medical teams sent to Hubei, and the number of COVID-19 cases among HCWs in local hospitals also significantly decreased, thereby indicating that hospital-acquired infections of SARS-CoV-2 among HCWs are fully preventable.
\end{abstract}

Keywords: COVID-19; infection control; healthcare-associated infections; healthcare workers; SARS-CoV-2

\section{Introduction}

As of 18th Apr. 2021, more than 140,000,000 cases of coronavirus disease 2019 (COVID-19) were reported in more than 192 countries [1]. Healthcare workers (HCWs) have the potential for direct or indirect exposure to COVID-19 patients or infectious materials [2]. HCWs infected with COVID-19 are a serious medical and social problem. An early report in China found that among 138 cases of COVID-19, HCWs accounted for $29 \%$ (40/138) [3]. Among 72,314 COVID-19 cases reported in mainland China as of February 11,2020 , the number of HCWs with confirmed COVID-19 was 1,716, accounting for 3.84\% of the confirmed cases [4]. Among 315,531 cases of COVID-19 reported by the Centers for Disease Control and Prevention (CDC; Atlanta, GA, USA), covering the period of February 12 to April 9, 2020, 49,370 cases were assessed to indicate their eligibility as a HCW, of whom 9,282 cases were identified as HCWs (19\%). Of 1,423 COVID-19-confirmed HCWs with data on the location of contact with confirmed cases, $780(55 \%)$ cases had a contact with a COVID-19 patient only in hospitals [2]. As a result, COVID-19 is relatively common 
among HCWs, both through community-acquired and hospital-acquired infections (HAIs). The presentation of symptoms provides an opportunity for case detection and isolation, facilitating the interruption of transmission. Because the majority of SARS-CoV patients had severe symptoms, they were relatively simple to identify and isolate, thereby interrupting the chain of transmission.

In the early stages of the outbreak of COVID-19 in Hubei, China, the local healthcare system was overwhelmed. A total of 29 provinces (autonomous regions and municipalities directly organized under the Central Government of the People's Republic of China) and the Xinjiang Production and Construction Corps sent several medical teams to Hubei, of which Jiangsu, Guangdong, Liaoning, Zhejiang, and Shandong provinces sent a large number of healthcare providers (HCPs), accounting for $28.69 \%$ of the total healthcare providers in China (refer to Table 1 for further details). Since January 27, 2020, China has dispatched 346 national medical teams with 42,632 HCPs (with participation of 4000 HCPs from military medical unit), including 10,517 physicians, 26,530 nurses, and 1,585 other personnel. There are 28,567 female cases, and male: female ratio is about 1 : 2.04. The ratio of male: female doctors is about 2.5: 1 , and the ratio of male: female nurses is about 1: 6.2 (refer to Table 2 for further details). A great number of HCWs who had no specialty in infectious disease were recruited to provide healthcare to patients with COVID-19 [5]. They cared for confirmed/suspected cases who experienced an increased risk of exposure to SARS-CoV-2 virus.

At a press conference held by the State Council Information Office of China on March 31, 2020, the minister of the National Health Commission, Ma Xiaowei, announced that owing to the strict implementation of prevention and control measures for HAIs, as a significant outcome, none of the $42,632 \mathrm{HCWs}$, who travelled across the Hubei province, had been infected with SARS-CoV-2 [6]. The present article aimed to discuss the prevention measures of SARS-CoV-2 infection among HCWs in Wuhan (China). The authors are the frontline physicians or infection control professionals who directly or indirectly provided prevention strategies for the 42,632 HCWs from across the country who were subjected to the risk of COVID-19.

\section{Viewpoint and Discussion}

2.1 General principles for the prevention of SARS-CoV-2 infections among HCWs

1 The timely detection and early diagnosis of COVID-19 can reduce the risk of infection among HCWs.

2 HCWs should be aware of the sources and routes of transmission for SARS-CoV-2 and should be informed that they are high-risk personnel because of their often extensive and close contact with COVID-19 cases in healthcare settings [7].

3 HCWs should be clearly aware of the key measures for the prevention of SARSCoV-2 infection.

4 HCWs should be trained for the proper use of personal protective equipment (PPE).

5 Healthcare institutions should assist HCWs with PPE according to the pre-defined standards.

6 Healthcare institutions need carry out infection surveillance on HCWs and introduce effective interventions.

7 Appropriate arrangements should be made for regulating HCWs' lifestyle to prevent cross-infection among hospitals, communities, and families.

8 Proper living arrangements should be made for HCWs to prevent cross-infection and enhance the body's immunity system.

9 HCWs should receive training modules on basic theory and skills required for preventing COVID-19.

10 Improve the organization of infection prevention and control, formulate standard operating procedures (SOPs), and carry out supervision and guidance.

\subsection{The early diagnosis of COVID-19 can reduce the risk of infection among HCWs}


It is noteworthy that the risk of infection is primarily concentrated in the triage areas, emergency departments, fever clinics, and among HCWs who have connection with the above-mentioned sites. Thus, HCWs in other areas of hospitals/medical centers, e.g. inpatient wards, should not be exposed to suspected/confirmed cases with COVID-19. HCWs involved in early diagnosis are strongly advised to take effective protective measures. Therefore, it is highly essential to establish triage stations staffed by registered nurses and physicians, who should immediately transfer patients with fever to the fever clinic [8], where samples are taken for nucleic acid amplification test in a timely manner. This may facilitate the early detection of suspected/confirmed cases with COVID-19. Additionally, a variety of dissemination materials, including brochures, flyers, factsheets, posters, etc., should be utilized to inform patients with acute fever who should be directly transferred to fever clinics [9].

Observation wards should be provided in areas connected to fever clinics, where assessment of suspected cases can be easily performed for the diagnosis and treatment. For suspected cases who are under observation, each case must be isolated in one room to prevent cross-infection. The timely admission of confirmed cases to isolation wards or transfer to designated hospitals as advised by local governments may effectively prevent cross-infection with other patients, while preventing exposure of other HCWs to infection, as well as reducing the risk of infection. In hospitals where conditions permit, COVID-19 patients should be admitted to negative pressure wards for isolation and treatment, especially patients who need to undergo aerosol-generating procedures, including tracheotomy, tracheal intubation, cardiopulmonary resuscitation, and open suctioning, which may further decrease the risk of infection among HCWs. Isolation wards must meet the pre-defined requirements, such as establishment of the three zones and two aisles, with buffer zones between the different zones (e.g., clean zone, potentially contaminated zone, contaminated zone, etc.).

\subsection{HCWs should be aware of sources and routes of transmission for SARS-CoV-2 and should be clearly informed that they are high-risk personnel}

To our surprise, $23 \%$ of those identified with SARS-CoV-2 infection, including those with abnormal chest computed tomography (CT) scan on admission, were completely asymptomatic throughout infection. It is highly essential to inform HCWs about sources of SARS-CoV-2 infection, including COVID-19 patients and asymptomatically infected individuals [10,11]. Individuals with asymptomatic COVID-19 infection present a unique challenge as they lack characteristics that might indicate they are infected. Asymptomatically infected cases, however, particularly those with no history of contact with a known SARS-CoV-2 patient, are hard to identify. There are several case reports of SARS-CoV-2 transmission from asymptomatically infected individuals [10,12], including a report of presumed transmission by asymptomatic carriers with normal chest CT scan [13].

Respiratory viruses replicate in the respiratory tract from where they are subsequently shed and transmitted via respiratory secretions. Respiratory viruses spread via three different transmission routes: contact (direct or indirect), droplet, and aerosol transmission. Contact transmission refers to direct virus transfer from an infected person to a susceptible individual (e.g. via contaminated hands) or indirect virus transfer via intermediate objects (fomites). Transmission of virus through the air can occur via droplets or aerosols [14,15]. The most common mode for spread of respiratory viruses is respiratory droplet transmission. Transmission of infection can also occur indirectly after the infected droplets have deposited if a host touches the contaminated surface and then touches their face. Airborne transmission occurs when virus-laden fine respiratory droplets remain viable in the environment and are inhaled by a susceptible individual. This transmission can occur either directly by inhalation of fine droplets expelled from an infected person or during aerosol-generating procedures on an infected individual. The personal protection of HCWs mainly aims to prevent the entrance of COVID-19 from the respiratory tract. 


\subsection{HCWs should be clearly aware of key measures to prevent infection}

Once HCWs are informed about the sources and routes of COVID-19 transmission, they may readily comprehend principles and key points of personal protection, and may consciously comply with requirements for protection, in addition to the proper use of protective equipment. On the basis of importance of adherence to standard precautions, as well as droplet, contact, and airborne isolation precautions, wearing mask and regular handwashing are crucial among all protective measures [14,16] . Surgical masks significantly reduce detection of influenza virus RNA in respiratory droplets and coronavirus RNA in aerosols, with a trend toward reduced detection of coronavirus RNA in respiratory droplets. The WHO advised the use of masks as a part of a comprehensive package of prevention and control measures to limit the spread of COVID-19. Contact transmission occurs when contaminated hands touch the mucosa of the mouth, nose, or eyes; the virus can also be transferred from one surface to another by contaminated hands, which facilitates indirect contact transmission. Consequently, hand hygiene is extremely important to prevent the spread of the COVID-19 virus.

Other protective measures mainly concentrate on preventing droplets or aerosols containing the SARS-CoV-2 virus from contaminated cloths, skin, shoes, etc. of HCWs, which in turn may cause droplet or airborne transmission. Most of the touchable surfaces in the designated hospital for COVID-19 are heavily contaminated, suggesting that the environment is a potential medium of disease transmission [17-20]. Thus, further strict environmental surface hygiene measures should be adopted to prevent HCWs from contacting with contaminated environmental surfaces, and hand hygiene should be promoted to prevent the spread of COVID-19.

\subsection{HCWs should be trained for individualized protective techniques and the correct use of PPE}

HCWs should receive training modules to understand the significance of PPE in the prevention of COVID-19, including masks, hand hygiene products, wearing of protective clothing (e.g., surgical gowns, isolation gowns, and coveralls), gloves, headgear, goggles, face shields, shoe covers, positive-pressure respirators, etc. HCWs should be informed about protective principles behind individualized protective techniques and PPE.

HCWs need learn techniques required for utilization of PPE to maximize the efficacy of such equipment. For instance, when wearing surgical masks, the mouth and nose must be fully covered according to the manufacturer's instructions, and nose-bridge wire must be pressed downwards with both hands simultaneously to ensure that the mask is fitted flush against the face. Respirators should be worn according to the manufacturer's instructions, with a special attention paid to the shaping of the mask to fit the bridge of the nose [21]. Fit testing confirms the fit of any respirator that forms a tight seal on a user's face before it is used in workplace. This ensures that users are receiving the expected level of protection by minimizing contaminant leakage into the face-piece. PPE should be donned and removed according to the recommended procedures, and a special attention should be paid to the techniques preventing the contamination of one's skin and clothing while removing the outer protective equipment. Masks should be removed last, and hand hygiene should be performed before they are taken off. The eyes should remain closed when removing goggles and the breath should be held while removing masks, to maximize the prevention of inhaling droplets or aerosols during removal.

\subsection{Healthcare institutions should assist HCWs with PPE that meet the required stand- ards [16]}

Healthcare institutions must assist HCWs with PPE that meet the required standards, and communication with suppliers of protective products must be strengthened to ensure that an appropriate reserve of PPE is available. In the event of emergency outbreaks, manufacturers must be able to guarantee a steady supply. During major epidemics, 
particularly in cases where a sharp increase in the demand for PPE within a short period of time is observed, an emergency response planning to reserve production capacity is required.

2.7 Fever and respiratory symptoms of HCWs should be monitored, and those who present with symptoms must be prohibited from working

During the epidemic, healthcare institutions, including medical teams, must carry out infection monitoring, including fever and respiratory symptoms, particularly on HCWs, and establish work systems and standard procedures. Responsible departments and personnel should be appointed to ensure the accountability of monitoring records. The purposes of monitoring are described as follows: first, to ensure timely detection of fever and respiratory symptoms among HCWs to enable early diagnosis and treatment; second, to prevent transmission or even outbreaks, caused by infections among HCWs [14]. Monitoring methods include daily self-reporting of fever, respiratory symptoms or other physical discomfort, daily measurement of body temperature, etc. If, during the COVID-19 epidemic, a HCW presents with fever and respiratory symptoms, the first step is to indicate whether he/she has been infected by SARS-CoV-2, followed by immediate isolation and testing for COVID-19. Otherwise, the infection may spread very quickly among HCWs, which leads to serious consequences and may affect patients, as well as causing an outbreak of hospital-acquired infections. Thus, it should be emphasized that if a HCW was infected by SARS-CoV-2, he/she must be isolated and treated immediately. Other HCWs who have had close contact with this HCW may need to be isolated for medical observation and nucleic acid testing to achieve early detection of infected individuals and to prevent the spread of infection. HCWs with fever and respiratory symptoms must immediately seek medical attention and rest. HCWs should be encouraged to care for each other and be promptly reminded not to work if they present with fever and respiratory symptoms.

\subsection{Proper living arrangements should be provided for HCWs to prevent cross-infec- tion and weakening of immunity system}

During the fight against the epidemic in Wuhan, Hubei, uniform living arrangements were provided for the national medical teams supporting Hubei and Wuhan. All teams lived in hotels, with one person in one room. All personnel were asked to wear surgical masks when leaving their rooms, maintain a distance of $1 \mathrm{~m}$ between each other, and closely adhere to cough etiquette. Good cleanliness and ventilation were maintained inside the rooms. Meals were provided as individual servings, and personnel returned to their separate rooms once they had collected their meals to minimize gatherings [22]. The medical teams' circle of activity was confined to two sites, hotel and hospital's isolation area, and the line connecting these two sites. HCWs were able to walk in open spaces around the hotel while wearing masks, and buses were arranged for longer distances of travel. Thus, a perfect closed-loop management was achieved for those medical teams. The hotel staff were relatively fixed and monitored for fever and respiratory symptoms.

The management of HCWs at local hospitals who worked in fever clinics and hospitals' isolation areas was also based on the above-mentioned policy. In principle, HCWs also had uniform living arrangements and did not return their homes when they were fighting against the pandemic. This not only prevented community-acquired infections from family clusters, but also prevented family clusters of iatrogenic infections.

\subsection{HCWs should receive training modules on basic theory and skills}

Theoretical and practical trainings are worthy for fighting against the pandemic. Theoretical training modules should contain basic information about COVID-19, including etiology, the three aspects of COVID-19 transmission (infection sources, infection routes, and susceptible persons), factors influencing infection control (awareness and conditions for infection control), clinical manifestations, diagnosis and differential diagnosis, 
isolation and treatment, and reporting and discharge criteria; the requirements of policies, laws, regulations and standards; and theories of nosocomial infection prevention and control, personal protection, etc. Practical training modules should mainly concentrate on the selection and use of PPE, the procedures for donning and removal, and precautions to note, especially for masks and hand hygiene [14,23,24]. Practical training modules should also include training for wearing and removing PPE, and assessments should be conducted case-by-case to determine whether the requirements are satisfied.

\subsection{Improving organization of infection prevention and control, formulating SOPs,} and performing supervision and guidance - success or failure hinges on details

Healthcare institutions should improve the organizational system for the prevention and control of nosocomial infections [14], and departments of infection control should be specifically responsible for implementation of SOPs, in addition to the formulation of relevant SOPs. Infection control supervisors should be appointed to medical teams or isolation areas, who could be full-time infection control staff, or physicians or nurses transferred from clinical departments. The primary roles of infection control supervisors are described as follows: First, infection control supervisors should ensure the implementation of SOPs, and success or failure can be determined by their attention to details; second, infection control supervisors are responsible for the practical training of medical teams' members. One-to-one training should be provided, and all personnel must pass the assessment; third, infection control supervisors are responsible for guiding and supervising the correct wearing and removal of PPE by medical teams' members in buffer zones, and for finding timely solutions for problems that arise. They also must ensure that PPE is worn according to requirements when entering contaminated zones and are removed correctly when exiting those zones; fourth, infection control supervisors must enter the contaminated zones to inspect and guide the prevention and control of nosocomial infections and the personal protection of HCWs, thereby ensuring that HCWs meet the specific requirements for PPE during working hours.

\section{Conclusions}

During fighting against COVID-19 in China, although a large number of HCWs were infected by SARS-CoV-2 in the early stages of the epidemic, the timely adoption of measures indicated that, a faster rate of diagnosis could be achieved, patients were isolated in-time, HCWs' safety was prioritized, training on basic protective knowledge and unified management of HCWs was strengthened, and effective protective measures were implemented. This resulted in the accomplishment of zero SARS-CoV-2 infection among the 42,632 members of the national medical teams sent to Hubei, and the number of COVID-19 cases among HCWs in local hospitals also significantly decreased, thereby indicating that HAIs of SARS-CoV-2 among HCWs are fully preventable.

Table 1. The number of medical teams and healthcare providers in different provinces in mainland China

\begin{tabular}{cccc}
\hline Provinces & $\begin{array}{c}\text { Number of medical } \\
\text { teams }\end{array}$ & $\begin{array}{c}\text { Number of healthcare } \\
\text { providers }\end{array}$ & $\begin{array}{c}\text { Number of female } \\
\text { healthcare providers }\end{array}$ \\
\hline Jiangsu & 21 & 2771 & 1655 \\
\hline Guangdong & 26 & 2478 & 1486 \\
\hline Liaoning & 11 & 2053 & 1495 \\
\hline Zhejiang & 16 & 1994 & 1086 \\
\hline Shandong & 14 & 1788 & 1093 \\
\hline Shanghai & 14 & 1634 &
\end{tabular}




\begin{tabular}{|c|c|c|c|}
\hline Provinces & $\begin{array}{c}\text { Number of medical } \\
\text { teams }\end{array}$ & $\begin{array}{c}\text { Number of healthcare } \\
\text { providers }\end{array}$ & $\begin{array}{c}\text { Number of female } \\
\text { healthcare providers }\end{array}$ \\
\hline Chongqing & 15 & 1618 & 1129 \\
\hline Heilongjiang & 10 & 1538 & 1102 \\
\hline Shanxi & 13 & 1511 & 1106 \\
\hline Hunan & 16 & 1458 & 975 \\
\hline Sichuan & 10 & 1457 & 919 \\
\hline Guizhou & 12 & 1405 & 902 \\
\hline Fujian & 12 & 1366 & 919 \\
\hline Anhui & 11 & 1324 & 863 \\
\hline Tianjin & 12 & 1292 & 856 \\
\hline Henan & 13 & 1262 & 717 \\
\hline Beijing & 22 & 1230 & 832 \\
\hline Jiangxi & 10 & 1226 & 829 \\
\hline Ji Lin & 14 & 1185 & 874 \\
\hline Yunnan & 7 & 1133 & 790 \\
\hline Hebei & 9 & 1091 & 684 \\
\hline Guangxi & 10 & 957 & 667 \\
\hline Shaanxi & 11 & 917 & 717 \\
\hline Hainan & 7 & 853 & 572 \\
\hline Inner Mongolia & 7 & 800 & 585 \\
\hline Ningxia & 7 & 782 & 592 \\
\hline Gansu & 6 & 777 & 590 \\
\hline Xinjiang & 3 & 386 & 302 \\
\hline Qinghai & 2 & 239 & 194 \\
\hline $\begin{array}{l}\text { Production and } \\
\text { construction corps }\end{array}$ & 2 & 107 & 49 \\
\hline $\begin{array}{c}\text { Military medical } \\
\text { unit }\end{array}$ & 3 & 4000 & 2500 \\
\hline subtotal & 346 & 42632 & 28566 \\
\hline
\end{tabular}

Table 2. Healthcare providers' sex ratio by occupational category

\begin{tabular}{ccccc}
\hline \multirow{2}{*}{ Occupational category } & $\begin{array}{c}\text { Number of healthcare } \\
\text { providers }\end{array}$ & \multicolumn{2}{c}{ Gender } & \multirow{2}{*}{ NT $^{*}$} \\
\cline { 3 - 4 } & 10517 & 7542 & 2975 & 0 \\
\hline Doctor & 26530 & 3697 & 22833 & 0 \\
\hline Nurse & 1585 & 1264 & 259 & 62 \\
\hline Others $^{*}$ & & & & Female \\
\hline
\end{tabular}




\begin{tabular}{ccccc}
\hline \multirow{2}{*}{ Occupational category } & $\begin{array}{c}\text { Number of healthcare } \\
\text { providers }\end{array}$ & \multicolumn{2}{c}{ Gender } & \multirow{2}{*}{ NT $^{*}$} \\
\cline { 3 - 4 } $\begin{array}{c}\text { Military medical } \\
\text { personnel }\end{array}$ & 4000 & 1500 & 2500 & 0 \\
\hline Total & 42632 & 14003 & 28567 & 62 \\
\hline
\end{tabular}

Others*: including medical technology, pharmaceutical personnel, disease prevention and control personnel, management personnel, and scientific researchers

$\mathrm{NT}^{* *}$ : The personnel did not fill the gap in gender.

\begin{abstract}
Author Contributions: AW, CL, and LL conceived and designed the project; AW, CL, and LL contributed to experience sharing and data assessment; CT and XJ performed the data and reference collection. XD provided the data of Tables 1 and 2, and carried out data analysis; AW, CT, and CL drafted the manuscript; AW and CL revised and re-edited the manuscript. All the authors read and approved the final version of the manuscript.
\end{abstract}

Funding: This study was financially supported by the COVID-19 Emergency Research Project of Hunan Province (Grant Nos. 2020SK3028 and 2020SK3027).

Conflicts of Interest: All the authors declare that there is no conflict of interest.

\title{
References
}

1. Medicine. JHU (2020) Coronavirus COVID-19 Global Cases by the Center for Systems Science and Engineering (CSSE) at Johns Hopkins University (JHU). . https://coronavirusjhuedu/maphtml [cited on 18 Apr 2021].

2. Team CC-R (2020) Characteristics of Health Care Personnel with COVID-19 - United States, February 12-April 9, 2020. MMWR Morb Mortal Wkly Rep 69: 477-481.

3. Wang D, Hu B, Hu C, Zhu F, Liu X, et al. (2020) Clinical Characteristics of 138 Hospitalized Patients With 2019 Novel CoronavirusInfected Pneumonia in Wuhan, China. JAMA.

4. Epidemiology Working Group for Ncip Epidemic Response CCfDC, Prevention (2020) [The epidemiological characteristics of an outbreak of 2019 novel coronavirus diseases (COVID-19) in China]. Zhonghua Liu Xing Bing Xue Za Zhi 41: 145-151.

5. China NHCotPsRo National Health Commission of the People's Republic of China Press conference of the joint prevention and control mechanism of the State Council on Feb 29, 2020. http://wwwnhcgovcn/xwzb/

webcontrollerdo?titleSeq=11248\&gecstype=1 (accessed July 10, 2020).

6. China TSCIOotPsRo (2020) SCIO briefing on progress of Central Guidance Team in guiding epidemic control and medical rescue on March 31. http://englishsciogovcn/pressroom/2020-04/03/content 75893890 Ohtm (accessed July 12, 2020).

7. CDC. Interim U.S. Guidance for Risk Assessment and Work Restrictions for Healthcare Personnel with Potential Exposure to COVID-19. . https://wwwcdcgov/coronavirus/2019-ncov/hcp/guidance-risk-assesment-hcphtml[cited on 12 July 2020$] 2020$.

8. Xiao Y, Tan C, Duan J, Wu A, Li C (2020) An effective model for the outpatient management of COVID-19. Infect Control Hosp Epidemiol: 1.

9. CDC. Standard Operating Procedure (SOP) for Triage of Suspected COVID-19 Patients in non-US Healthcare Settings: Early Identification and Prevention of Transmission during Triage. https://wwwcdcgov/coronavirus/2019-ncov/hcp/non-us-settings/soptriage-prevent-transmissionhtml(accessed July 14, 2020).

10. Pan X, Chen D, Xia Y, Wu X, Li T, et al. (2020) Asymptomatic cases in a family cluster with SARS-CoV-2 infection. Lancet Infect Dis 20: 410-411.

11. Furukawa NW, Brooks JT, Sobel J (2020) Evidence Supporting Transmission of Severe Acute Respiratory Syndrome Coronavirus 2 While Presymptomatic or Asymptomatic. Emerg Infect Dis 26. 
12. Yu P, Zhu J, Zhang Z, Han Y (2020) A Familial Cluster of Infection Associated With the 2019 Novel Coronavirus Indicating Possible Person-to-Person Transmission During the Incubation Period. J Infect Dis 221: 1757-1761.

13. Bai Y, Yao L, Wei T, Tian F, Jin DY, et al. (2020) Presumed Asymptomatic Carrier Transmission of COVID-19. JAMA 323: 14061407.

14. WU An-hua HX, LI Chun-hui, et al. Novel coronavirus (2019-nCoV) pneumonia in medical institutions: problems in prevention and control[J]. . Chin J Infect Control, 2020, 19(2): 99-104

15. China. NHCotPsRo Guidelines on diagnosis and treatment of novel coronavirus pneumonia (Trial sixth edition)[J]. . ChinJ Infect Control, 2020, 19(2): 192-195

DOI: 1012138/jissn1671-963820206154.

16. China. NHCotPsRo Technical guidelines on prevention and control of novel coronavirus infection in medical institutions (First edition)[J]. . Chin J Infect Control, 2020, 19(2): 189-191 DOI: 1012138/jissn1671-963820206152.

17. Guo ZD, Wang ZY, Zhang SF, Li X, Li L, et al. (2020) Aerosol and Surface Distribution of Severe Acute Respiratory Syndrome Coronavirus 2 in Hospital Wards, Wuhan, China, 2020. Emerg Infect Dis 26: 1583-1591.

18. Ong SWX, Tan YK, Chia PY, Lee TH, Ng OT, et al. (2020) Air, Surface Environmental, and Personal Protective Equipment Contamination by Severe Acute Respiratory Syndrome Coronavirus 2 (SARS-CoV-2) From a Symptomatic Patient. JAMA.

19. Wang H, Mo P, Li G, Chen P, Liu J, et al. (2020) Environmental virus surveillance in the isolation ward of COVID-19. J Hosp Infect 105: 373-374.

20. Ye G, Lin H, Chen S, Wang S, Zeng Z, et al. (2020) Environmental contamination of SARS-CoV-2 in healthcare premises. J Infect.

21. Coffey CC, Lawrence RB, Campbell DL, Zhuang Z, Calvert CA, et al. (2004) Fitting characteristics of eighteen N95 filteringfacepiece respirators. J Occup Environ Hyg 1: 262-271.

22. LI Liu-yi WA-h, YAO Xi. Prevention and control of novel coronavirus pneumonia among health care workers in resident of medical team. . Chin J Infect Control, 2020, 19(2): 123-125 DOI: 1012138/jissn1671-963820205364.

23. CDC. Interim Infection Prevention and Control Recommendations for Patients with Suspected or Confirmed Coronavirus Disease 2019 (COVID-19) in Healthcare Settings. . https://wwwcdcgov/coronavirus/2019-ncov/hcp/infection-control-recommendationshtml [cited on 12 July 2020].

24. WHO. (2020) Infection prevention and control during health care when novel coronavirus (nCoV) infection is suspected(Interim guidance). . https://wwwwhoint/publications-detail/infection-prevention-and-control-during-health-care-when-novel-coronavirus(ncov)-infection-is-suspected-20200125[cited on 12 July 2020]. 